\title{
TORTELLA FASCICULATA AND T. PSEUDOFRAGILIS (POTTIACEAE, BRYOPHYTA) IN HUNGARY
}

\author{
Peter Erzberger ${ }^{1} \&$ Beáta PAPP ${ }^{2}$ \\ ${ }^{1}$ Belziger Str.37,D-10823 Berlin, Germany; erzberger.peter@gmail.com \\ ${ }^{2}$ Department of Botany, Hungarian Natural History Museum, H-1431 Budapest, Pf. 137, Hungary
}

Erberger, P. \& Papp, B. (2018): Tortella fasciculata and T. pseudofragilis (Pottiaceae, Bryophyta) in Hungary. - Studia bot.hung. 49(2): 39-48.

\begin{abstract}
Revision of specimens housed in the bryophyte collection of the Hungarian Natural History Museum, Budapest (BP) and recent collections of the first author as well as of other bryologists enabled the establishment of the distribution of Tortella fasciculata (Culm.) Culm. and T. pseudofragilis (Thér.) Köckinger \& Hedenäs in Hungary, two recently described segregates of the obsolete name T. bambergeri auct. eur. non (Schimp.) Broth. T. fasciculata proved to be widespread in the country, occurring in 14 geographic regions: Aggtelek Karst, Bükk Mts, Börzsöny Mts, Visegrád Mts, Pilis Mts, Buda Mts, Gerecse Mts, Vértes Mts, Bakony Mts, Balaton Uplands, Keszthely Mts, Köszeg Mts, Mecsek Mts, and Geresdi dombság. It grows on average at an elevation of $402 \pm$ $33.2 \mathrm{~m}$ a. s. 1 . (mean \pm standard error). T. pseudofragilis, on the other hand, is restricted to 4 regions: Aggtelek Karst, Bükk Mts, Pilis Mts, and Bakony Mts, and grows at significantly higher elevations of $647 \pm 47.5 \mathrm{~m}$ a.s.l. Distribution maps, a key for Hungarian Tortella species, and an illustration of the most important microscopic feature for the discrimination of the two segregates are also presented.
\end{abstract}

Key words: distribution maps, identification key, marginal leaf cells, revision, Tortella bambergeri

\section{INTRODUCTION}

Integrative taxonomy combining molecular and morphological methods has proved to be a powerful tool for the disentangling of a group of closely related taxa, e.g. in the Ulota crispa complex (CAPARRós et al. 2016). Recently, another example has been given by KöCKINGER \& HEDENÄs (2017). They showed that Tortella bambergeri (Schimp.) Broth., as understood by European bryologists over the last decades, consists in fact of two distinct species, Tortella fasciculata (Culm.) Culm. and T. pseudofragilis (Thér.) Köckinger \& Hedenäs, with different geographical and ecological affinities: the thermophilic T. fasciculata is characterized as suboceanic-sub-Mediterranean, whereas the moderately cryophilic $T$. pseudofragilis is a boreo-montane species.

The name $T$. bambergeri used traditionally cannot be applied any longer since the type of Trichostomum bambergeri Schimp., the basionym of Tortella 
bambergeri, proved to belong to the variable and widespread T. tortuosa (Hedw.) Limpr. (KöCKINGER \& HEDENÄS 2017).

In Hungary, $T$. bambergeri auct. has for a long time been a neglected taxon, which was added to the country's list only recently (ERZBERGER \& PAPP 2004) based on specimens in JE collected by R. Marstaller (MARSTALLER 1993). Although it has been collected afterwards a few times, it is certainly under-recorded due to its similarity with the frequent $T$. tortuosa.

KöCKINGER \& HEDENÄs (2017) present evidence of the occurrence of $T$. fasciculata in Hungary, quoting among the specimens studied with molecular methods a collection of Á. Boros from 1936, Hungary ('no locality provided'). $T$. pseudofragilis, the other segregate of $T$. bambergeri, has been found recently in the Bükk Mts (CASPARI \& ERZBERgER 2019).

The aim of the present paper is to study the distribution of the T. bambergeri segregates in Hungary, based on the revision of herbarium specimens and recent collections.

\section{MATERIAL AND METHODS}

T. bambergeri auct. specimens deposited in the bryophyte herbarium of the Hungarian Natural History Museum, Budapest (BP) were revised and all specimens originally labelled Tortella tortuosa (Hedw.) Limpr. or related taxa (T. niti$d a$ (Lindb.) Broth., T. viridiflava (De Not.) Broth.) collected in Hungary were screened (by B.P.) for characters of T. bambergeri auct. (fragile leaves, dull, not shiny leaf tips), resulting in 40 specimens which were then evaluated according to the description in KöCKINGER \& HEDENÄs (2017).

In addition, $T$. bambergeri auct. collected during the ongoing recording project (ERZBERger 2012, ERzBERger \& NÉMETh 2013), as well as selected specimens of the private herbaria of J. Nagy and of Cs. Németh were revised (by P. E.). Drawings were made with a Leitz drawing apparatus. Distribution maps were prepared according to the Central European mapping scheme (KIRÁLY et al. 2003, BARTHA et al. 2015).

Nomenclature of bryophytes follows PAPP et al. (2010).

\section{RESULTS AND DISCUSSION}

The molecular evidence for splitting Tortella bambergeri auct. and morphological descriptions of the two segregates are presented in great detail by KöCKINGER \& HEDENÄs (2017), which we will therefore not repeat here. We would like to emphasize the usefulness of the descriptions and illustrations in 
that paper which enabled us to apply the new concept to the Hungarian material without any difficulties.

Since there are now four instead of three species of Tortella known from Hungary, we present a key and an illustration of the microscopic key feature, the shape of the marginal cells (Fig. 1).

\section{Key to Tortella species in Hungary}

1a Cells of ventral surface of costa linear, smooth, not covered by isodiametric papillose laminal cells; leaf apex broadly acute, cucullate

T. inclinata

1b Ventral surface of costa covered by isodiametric papillose laminal cells, at least in upper part of leaf; leaves tapering to long, narrowly lanceolate apex; leaves \pm undulate when moist, much contorted when dry 2

2a Plants to $5 \mathrm{~cm}$ tall; leaves sometimes fragile; cells on dorsal surface of costa near leaf apex always elongate and mostly smooth (stereids), rarely papillose; stem mostly without central strand; peristome teeth twisted 1.5-3 times to the left T. tortuosa

2b Plants usually smaller; leaves fragile with most tips broken off transversely; cells on dorsal surface of costa near intact leaf apex quadrate and papillose; stem mostly with central strand; peristome teeth twisted $1 / 2$ turn to the left

3a Leaves when dry curled up to the apex, leaf tip often like a cork-screw; individual shoots of cushion well discernible from above and shaped like a ball of wool; marginal cells transversely elongate (wider than long) or quadrate, with hemispherically bulging outer walls causing the margin to be neatly crenulate; thermophilic plants growing at low elevations ... T. fasciculata

3b Dry leaves straight or slightly flexuose in their apical third and mostly oriented perpendicular to the shoot axis ('lying on the cushion surface'); individual shoots of cushion thereby less well discernible from above; marginal cells elongate (longer than wide) or quadrate, outer walls only slightly bulging; cryophilic plants of high elevations T. pseudofragilis
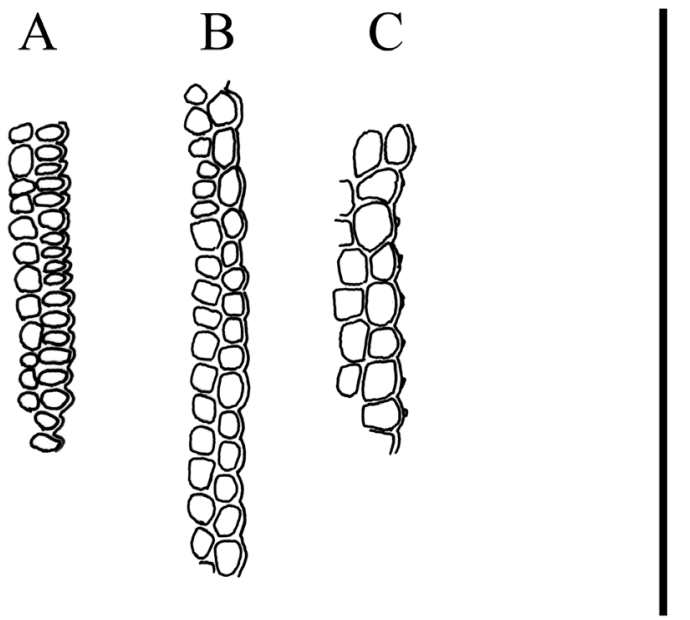

Fig. 1. Marginal cells of Tortella fasciculata and T. pseudofragilis: A = Tortella fasciculata; B, C = T. pseudofragilis (Scale bar: $200 \mu \mathrm{m}$ [A: Erzberger 7018, B, C: Erzberger 24387]) 
Although a central strand is mostly present in $T$. fasciculata and $T$. pseudofragilis, there are plants of both species where the central strand is lacking or indistinct. In Tortella tortuosa, on the other hand, the central strand is usually absent, but the presence of a central strand is reported in particular in alpine and subarctic morphs (KöCKINGER \& HEDENÄs 2017). Therefore, the presence or absence of a central strand should no longer be used as the key feature in the discrimination between $T$. tortuos $a$ and the T. bambergeri auct. segregates, as has been long-standing practice (e.g. Ahrens 2000, Frey et al. 2006).

Of the 67 specimens revised in this paper, 50 (including duplicates) proved to be $T$. fasciculata, collected in 33 different sites in $24 \mathrm{KEF}$-quadrats, whereas 17 specimens (including duplicates) from 12 different sites in $10 \mathrm{KEF}$-quadrats turned out to belong to T. pseudofragilis (see Appendix for specimen details). $T$. fasciculata clearly is more frequent in Hungary than T. pseudofragilis.

T. fasciculata occurs in the following 14 geographic regions of Hungary (modified after Boros 1968): Aggtelek Karst, Bükk Mts, Börzsöny Mts, Visegrád Mts, Pilis Mts, Buda Mts, Gerecse Mts, Vértes Mts, Bakony Mts, Balaton Uplands, Keszthely Mts, Köszeg Mts, Mecsek Mts, and Geresdi dombság (Fig. 2).

T. pseudofragilis, on the other hand, was found in 4 regions: Aggtelek Karst, Bükk Mts, Pilis Mts, and Bakony Mts (Fig. 3).

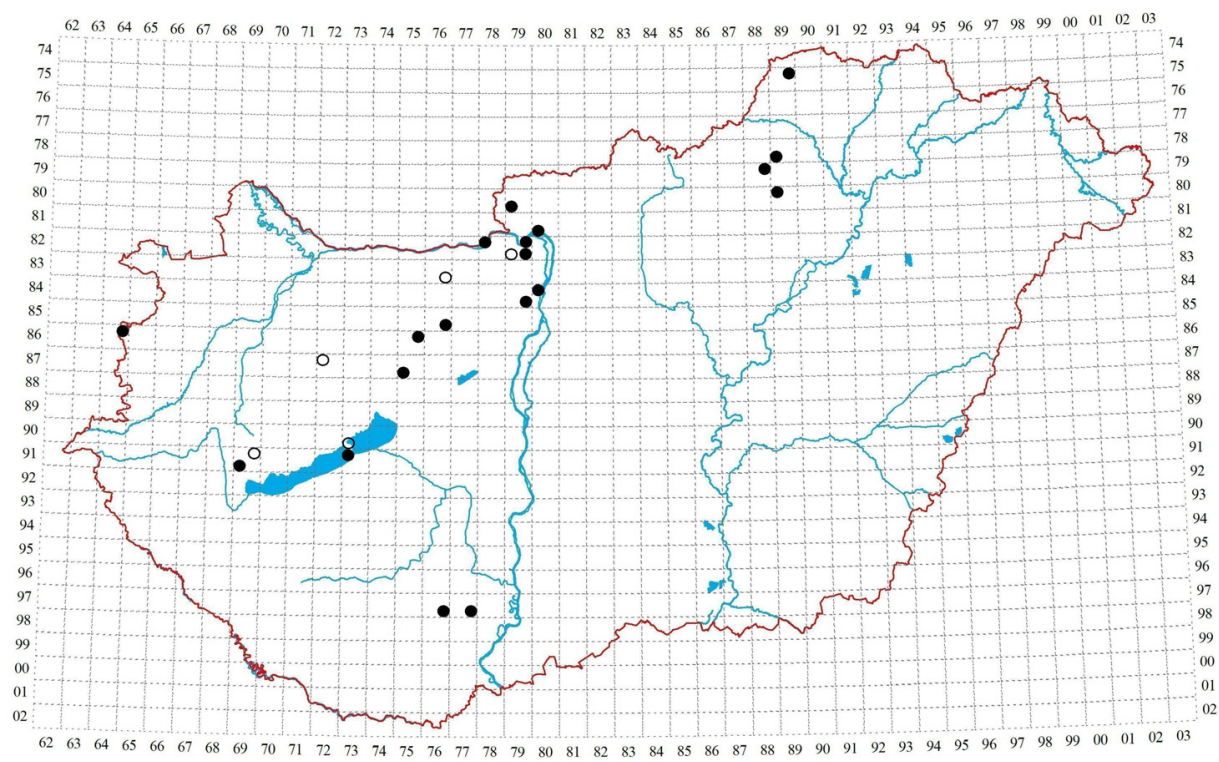

Fig. 2. Records of Tortella fasciculata. Open circles denote records before 1974, closed circles after that year. When both old and recent collections existed in the same grid cell, only the latter are shown. 
In all of these regions, where T. pseudofragilis was found, T. fasciculata occurs too. There are $4 \mathrm{KEF}$ quadrats with both species, and even one site, where both species occur together: the limestone cliff Fekete-kő near Pilisszentlélek (8279.3) in the Pilis Mts. That the two species can grow together in spite of their slightly different ecological profile is also reported by KöCKINGER \& HEDENÄs (2017) who even publish a photograph of a mixed stand.

Fortunately, most collection labels include data on elevation. It is thus possible to evaluate the average altitude of the two species in Hungary. T. fasciculata grows between 153 and $900 \mathrm{~m}$ a. s. 1 . (mean \pm standard error: $402 \pm 33.2$ ), whereas $T$. pseudofragilis grows on average at significantly higher elevations between 350 and $890 \mathrm{~m}$ a.s.l. (mean \pm standard error: $647 \pm 47.5$ ). This is in agreement with the altitudinal ranges reported by KöcKINGER \& HEDENÄs (2017) (lowland to $1200 \mathrm{~m}$ for T. fasciculata, 400 to 2500 for T. pseudofragilis) and their characterization of $T$. fasciculata as thermophilic, in contrast to $T$. pseudofragilis as moderately cryophilic.

According to our results, $T$. fasciculata seems to be less restrictive with respect to bedrock than T. pseudofragilis, which was found exclusively on limestone and dolomite rocks. $T$. fasciculata also grows predominantly on these substrates, but additionally occurs on (base-rich) siliceous rocks, e.g. andesite, dacite, phyllite, basalt, diabase, slate, calcareous sandstone.

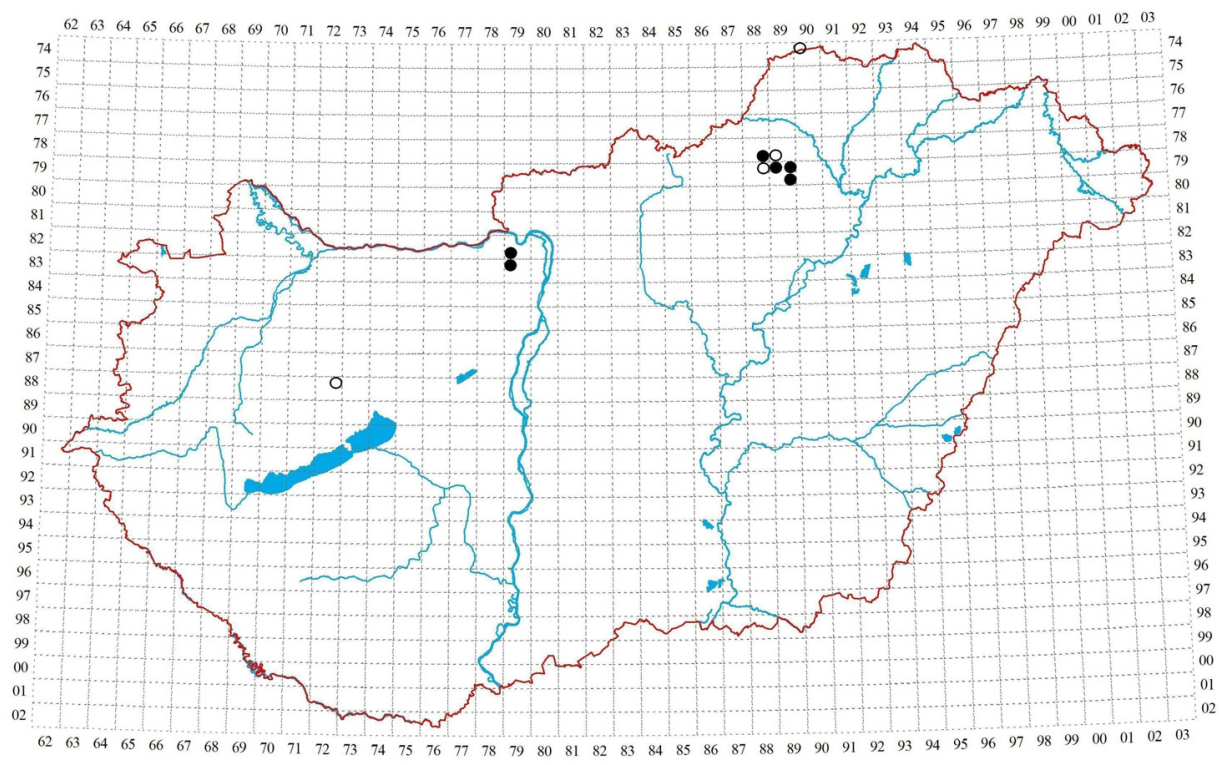

Fig. 3. Records of Tortella pseudofragilis. Open circles denote records before 1974, closed circles after that year. When both old and recent collections existed in the same grid cell, only the latter are shown. 
The results presented here are based on the ongoing recording activity and field work as well as the revision of the specimens housed in the bryophyte collection of the Hungarian Natural History Museum in Budapest. This is another example demonstrating the importance of collecting voucher specimens and preserving them in herbaria, even of seemingly ordinary species like $T$. tortuosa. Revision of such specimens allowed us to immediately establish the distribution of the newly described taxa.

Acknowledgements - We express our sincere thanks to all co-workers of the bryophyte recording project who participated in the collection of specimens evaluated in this paper: K. Baráth, S. Caspari, J. Csiky, J. Deme, D. Kovács, J. Nagy, Cs. Németh, P. Ódor, A. Schmotzer, E. Szurdoki, I.Zs. Tóth, W. Wiehle. To Cs. Németh we are indebted for the preparation of the distribution maps.

Összefoglaló: A Magyar Természettudományi Múzeum Mohagyủjteményében található, valamint az első szerző és más mohászok által az elmúlt években gyűjtött anyagok revíziója során sikerült tisztázni a Tortella fasciculata (Culm.) Culm. és a T. pseudofragilis (Thér.) Köckinger \& Hedenäs magyarországi elterjedését. Ezt a két fajt a korábban T. bambergeri auct. eur. non (Schimp.) Broth.-nek tartott taxonból, annak megszüntetésével, különítették el. A T. fasciculata szélesen elterjedt az egész országban, 14 földrajzi régióban van jelen: az Aggteleki-karszt, a Bükk, a Börzsöny, a Visegrádi-hegység, a Pilis, a Budai-hegység, a Gerecse, a Vértes, a Bakony, a Balaton-felvidék, a Keszthelyi-hegység, a Kőszegi-hegység, a Mecsek és a Geresdi-dombság területén. Az előfordulásainak átlagos tengerszint feletti magassága 402 $\pm 33,2 \mathrm{~m}$ (átlag \pm standard hiba). A T. pseudofragilis-t, viszont csak 4 régióból mutattuk ki: az Aggteleki-karsztról, a Bükkből, a Pilisből és a Bakonyból. Szignifikánsan magasabb tengerszintfeletti magasságon fordul elö, $647 \pm 47,5$ m-en. Jelen publikációban közöljük a két faj magyarországi elterjedési térképét, új határozó kulcsot adunk a magyarországi Tortella fajokhoz és a legfontosabb mikroszkopikus elkülönítő bélyegeket egy illusztráción is mutatjuk be.

\section{REFERENCES}

Ahrens M. (2000): Pottiaceae. - In: Nebel M. \& Philippi G. (eds): Die Moose Baden-Württembergs. Ulmer, Stuttgart. Vol. 2, 260-370 pp.

Bartha D., Király G., Schmidt D., Tiborcz V., Barina Z., Csiky J., Jakab G., Lesku B., Schmotzer A., Vidéki R., Vojt kó A. \& Zólyomi Sz. (eds) (2015): Magyarország edényes növényfajainak elterjedési atlasza. (Distribution atlas of vascular plants of Hungary). University of West Hungary Press, Sopron, $330 \mathrm{pp}$.

Boros Á. (1968): Bryogeographie und Bryoflora Ungarns. - Akadémiai Kiadó, Budapest, 466 pp.

Caparrós R., Lara F., Draper I., MAZimpaka V. \& Garilleti R. (2016): Integrative taxonomy sheds light on an old problem: the Ulota crispa complex (Orthotrichaceae, Musci). - Bot. J. Linn. Soc. 180: 427-451. https://doi.org/10.1111/boj.12397

Caspari S. \& ERzberger P. (2019): 54. Tortella pseudofragilis (Thér.) Köckinger \& Hedenäs [Hungary]. In: ELLIS, L. T. (ed.): New national and regional bryophyte records, 58. - J. Bryol. (in press). 
DieRSSEN K. (2001): Distribution, ecological amplitude and phytosociological characterization of European bryophytes. - Bryophytorum Bibliotheca 56: 1-289.

ERzBerger P. (2012): Project plan: bryophyte mapping of Hungary. - Programm and Abstracts, 8th Conference of European Committee for Conservation of Bryophytes, Budapest, 18-21 April 2012, p. 12.

Erzberger P. \& Németh Cs. (2013): Mohatérképezés Magyarországon - erdemények az iniciális fázisból. [Bryophyte mapping in Hungary - results from the initial phase]. - Absztraktkötet, II. Aktuális eredmények a kriptogám növények kutatásában konferencia, Eger, p. 13.

ERzBerger P. \& PAPP B. (2004): Annotated checklist of Hungarian bryophytes. - Studia bot. hung. 35: 91-149.

FREY W., FRAHM J.-P., Fischer E. \& LOBIN W. (2006): The liverworts, mosses and ferns of Europe. English edition revised and edited by T. L. Blockeel. - Harley Books, Colchester, 512 pp.

Király G., Balogh L., Barina Z., Bartha D., Bauer N., Bodonczi L., Dancza I., Farkas S., Galambos I., Gulyás G., Molnár V. A., Nagy J., Pifkó D., Schmotzer A., SomLYAY L., SZmorad F., Vidéki R., Vojtkó A. \& Zólyomi Sz. (2003): A magyarországi flóratérképezés módszertani alapjai. Útmutató és magyarázat a hálótérképezési adatlapok használatához. - Flora Pannonica 1(1): 3-20.

KöCKinger H. \& Hedenäs L. (2017): A farewell to Tortella bambergeri (Pottiaceae) as understood over the last decades. - J. Bryol. 39: 213-225. https://doi.org/10.1080/03736687.2017.1307313

Marstaller R. (1993): Die Moosgesellschaften des Villányer Gebirges in Südungarn. - Phytocoenologia 22(2): 193-273. https://doi.org/10.1127/phyto/22/1993/193

Papp B., Erzberger P., Ódor P., Hock Zs., Szövényi P., Szurdoki E. \& Tóth Z. (2010): Updated checklist and red list of Hungarian bryophytes. - Studia bot. bung. 41: 31-59.

(submitted: 09.11.2018, accepted: 29.11.2018)

\section{Appendix}

Specimens of Tortella fasciculata and T. pseudofragilis collected in Hungary

The following items are given: Geographic region in Hungary, grid cell according to the Central European mapping scheme (KIRÁLY et al. 2003, BARTHA et al. 2015), original text of label normally giving county, habitat, location and toponym, geographical coordinates (WGS 84), elevation above sea level (approximate), date of collection and name of collector(s), inventory number in BP or other herbarium.

Abbreviations: conf. $=$ determination confirmed by; det. = identified by, dupl. = duplicate; hb. = herbarium of; leg. = collected by; rev. = revised by; s.a. = without year, s.n. = without number, soc. $=$ associated bryophytes.

\section{Tortella fasciculata (Culm.) Culm.}

Aggtelek Karst 7589.2 Comit. Borsod-Abaúj-Zemplén. In rupibus calcareis supra vallem Jósva-völgy contra vallem Almás-völgy, 220 m a.s.l., 17.08.1988 leg. M. Rajczy, det. B. Papp as Tortella tortuosa (Hedw.) Limpr. (BP 165398). Bükk Mts 7889.3 Hungaria bor. com Borsod. In rupibus calcareis umbrosis montis Nagy Hetemér (Szentléleki romok) prope pag. Ómassa, $650 \mathrm{~m}$ 
a.s.l., 10.04.1950 leg. L. Vajda as Tortella nitida (Lindb.) Broth. (BP 103977). Borsod-Abaúj-Zemplén County, Örvénykő rock at Mályinka, on limestone rocks, N 4807'49.2”, E 20³2'17.5”, $790 \mathrm{~m}$ a.s.l., 02.06.2008 leg. B. Papp as Tortella tortuosa (Hedw.) Limpr. (BP 176460). 7988.2 Comit. Borsod. In rupibus calcar. silvat. montis Vöröskő-bérc prope Répáshuta, versus Szilvásvárad, $800 \mathrm{~m}$ a.s.l., 19.09.1937 leg. Á. Boros as Tortella tortuosa (Hedw.) Limpr. (BP 104159). Comit. Borsod. In rupibus diabas. sept. silvat. montis Nagy István erőse prope Nagyvisnyó, 900 m a.s.l., 30.09.1950 leg. Á. Boros as Tortella tortuosa (Hedw.) Limpr. (BP 104152). Borsod-Abaúj-Zemplén County, Répáshuta, Malom-hegy, N 4803'09.2”, E 020²8’22.8”, 630 m a.s.l., 14.04.2018 leg. P. Erzberger, J. Csiky, J. Deme \& A. Schmotzer (B-Erzberger 24474). 8089.1 Borsod-Abaúj-Zemplén County,

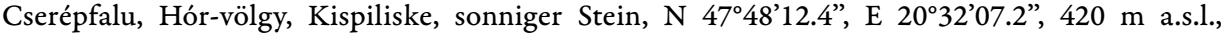
18.06.2012 leg. P. Erzberger, B. Papp \& P. Ódor (B-Erzberger 15333). Börzsöny 8079.3 Pest County, Nagybörzsöny, Zálog-bérc, árnyas andezit szikla, N 4755’21.4”, E $018^{\circ} 53^{\prime} 30.0^{\prime \prime}, 510 \mathrm{~m}$ a.s.l. and andezit sziklaüreg, $\mathrm{N} 47^{\circ} 55^{\prime} 25.8^{\prime \prime}$, E018 ${ }^{\circ} 53^{\prime} 33.1^{\prime \prime}, 525 \mathrm{~m}$ a.s.l., 23.06.2018 leg. et det. J. Nagy, conf. P. Erzberger (hb. J. Nagy s.n.). 8180.3 Pest County, Kismaros: Kalló-hegy, dácit sziklán, N 4750’00.7”, E 1900'59.1", 200 m a.s.l., 01.11.2017 leg. J. Nagy (hb. J. Nagy s.n., dupl. B-Erzberger s.n.). 8279.2 Pest County, Nagymaros, Szürke-hegy, löszös, töredezett andeziten, N 47 $46^{\prime} 12.8^{\prime \prime}$, E $18^{\circ} 56^{\prime} 18.8^{\prime \prime}$,

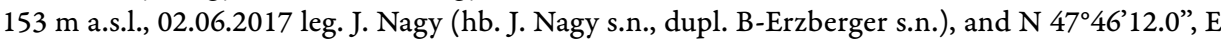
$18^{\circ} 56^{\prime} 12.0^{\prime \prime}, 155$ m a.s.l., 09.06.2017 leg. J. Nagy, det. P. Erzberger (BP 192515). Visegrád Mts 8279.4 Pest County, Pilisszentlászló, Kis-Disznó-hegy, on base-rich andesite rock, N $47^{\circ} 44^{\prime} 11.1^{\prime \prime}, \mathrm{E}$ $018^{\circ} 56^{\prime} 40.0^{\prime \prime}, 370 \mathrm{~m}$ a.s.l., 30.05.2014 leg. P. Erzberger \& B. Papp (B-Erzberger 17850a). Pilis Mts 8278.1 Komárom-Esztergom County, Strázsa Mt at Esztergom, around Sátorkőpuszta cave, lime-

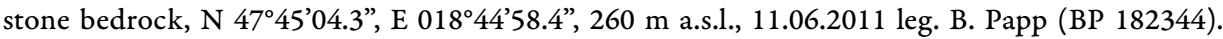
8279.3 Comit. Esztergom. In rupibus calcareis Fekete-kö prope Pilisszentlélek, 500-600 m a.s.l., 14.04.1946 leg. Á. Boros as Tortella nitida (Lindb.) Broth., rev. by P. Erzberger February 2001 as Tortella tortuosa (Hedw.) Limpr. (BP 2982; 2983 leg. 19.05.1946). Comit. Esztergom. In rupibus calcareis Fekete-kő prope Pilisszentlélek, 500-600 m a.s.l., 14.04.1946 leg. Á. Boros as Tortella nitida (Lindb.) Broth. (BP 103981; 103982 leg. 19.05.1946). Buda Mts 8479.4 Budapest, János hill, on

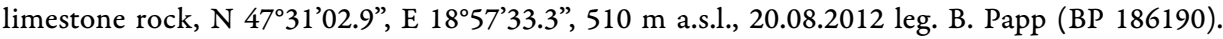
8480.1 Budapest, Hármashatár-hegy/Tábor-hegy, on limestone, N 47³3’28.0”, E 1900’07,1", 390 m a.s.l., 17.03.2014 leg. P. Erzberger (B-Erzberger 17069). Gerecse Mts 8376.4 Comit. Komárom. In rupibus calcar. orient. montis Peskő prope pag. Tarján, $350 \mathrm{~m}$ a.s.l., 25.04.1948 leg. Á. Boros as Tortella tortuosa (Hedw.) Limpr. (BP 104067). Vértes Mts 8576.4 Fejér megye; Csákvár; Kotlóhegy; félárnyékos dolomitsziklán, $\mathrm{N} 47.42141^{\circ}, \mathrm{E} 18.44177^{\circ}, 342 \mathrm{~m}$ a.s.l. and $\mathrm{N} 47.42348^{\circ}, \mathrm{E}$ $18.44421^{\circ}, 317$ m a.s.l., 04.05.2013 leg. Cs. Németh (hb. Németh 4838, 4842/1, dupl. BP 186728). 8675.2 Comit. Fejér. In rupibus dolomiticis supra vallem Csatorna-völgy prope Csákberény, 350$400 \mathrm{~m}$ a.s.l., 26.04.1936 leg. Á. Boros as Tortella nitida (Lindb.) Broth. (BP 103976). Hungaria centr. com. Fejér. In rupibus calcareis umbrosis vallis Csatornavölgy prope pag. Csákberény, 17.04.1949 leg. L. Vajda as Tortella nitida (Lindb.) Broth., rev. by B. Papp February 2001 as Tortella tortuosa (Hedw.) Limpr. (BP 2981). Comit. Fejér. In rupibus dolomit. silvat. vallis Csatorna-völgy prope Csákberény, 300-400 m a.s.l., 17.04.1949 leg. Á. Boros as Tortella nitida (Lindb.) Broth. (BP 103975). Comit. Fejér. In rupestribus dolomit. Cservágás prope pagum Csókakő, $400 \mathrm{~m}$ a.s.l., 20.08.1948 leg. Á. Boros as Tortella nitida (Lindb.) Broth., rev. by B. Papp February 2001 as Tortella tortuosa (Hedw.) Limpr. (BP 2985). Comit. Fejér. In rupestribus dolomit. Cservágás prope pagum Csókakő, $400 \mathrm{~m}$ a.s.l., 20.08.1948 leg. Á. Boros as Tortella nitida (Lindb.) Broth. (BP 103969). Comit. Fejér. In rupibus dolomit. sept. Papirtás prope pag. Csákberény, 300-400 m a.s.l., 21.08.1948 leg. Á. Boros as Tortella nitida (Lindb.) Broth., rev. by B. Papp February 2001 as Tortella tortuosa (Hedw.) Limpr. (BP 2984). Comit. Fejér. In rupibus dolomit. silv. Pap-irtás prope Csákberény, 300$400 \mathrm{~m}$ a.s.l., 21.08.1948 leg. Á. Boros as Tortella nitida (Lindb.) Broth. (BP 103971, 103973; 103974 
leg. 18.04.1949). Fejér County, Csókakő, Vár-völgy, castle ruin and surroundings, forest, shaded and exposed limestone/dolomite rock, calcareous grassland, 23.04.2001 leg. P. Erzberger (B-Erzberger 7018). Fejér County, Vértes Mts, Mór, Csóka-hill, on limestone grassland, N $47^{\circ} 22^{\prime} 08.8^{\prime \prime}, \mathrm{E}$ $18^{\circ} 15^{\prime} 13.0 ”, 410 \mathrm{~m}$ a.s.l., 22.03.2014leg. B. Papp (BP 189090). Bakony Mts 8772.1 Comit. Veszprém. In rupibus calc. silvat. ad Odvaskő vallis Szarvadárok prope Bakonybél, $300 \mathrm{~m}$ a.s.l., 03.10.1937 leg. Á. Boros as Tortella tortuosa (Hedw.) Limpr. (BP 104026). 8775.3 Comit. Fejér. In rupibus dolomit. sept. montis Baglyas-hegy prope Inota, 200-300 m a.s.l., 06.04.1951 leg. Á. Boros as Tortella nitida (Lindb.) Broth. (BP 103967). Comit. Fehér. In rupibus calcareis umbrosis montis Baglyas hegy supra vall. Hideg völgy prope pag. Inota, 28.03.1954 leg. L. Vajda as Tortella nitida (Lindb.) Broth., rev. by B. Papp February 2001 as Tortella tortuosa (Hedw.) Limpr. (BP 26939, 27015). Comit. Fejér. In rupibus dolomit. partis sept. montis Baglyas-hegy prope Inota, 250-300 m a.s.l., 28.03.1954 leg. Á. Boros as Tortella nitida (Lindb.) Broth. (BP 103968). Fejér County, Bakonykúti, Baglyas-hegy, exposed dolomite rock, $\mathrm{N} 47^{\circ} 14^{\prime} 18.0^{\prime \prime}, \mathrm{E} 18^{\circ} 11^{\prime} 40.6^{\prime \prime}, 200 \mathrm{~m}$ a.s.l., 21.04.2013 leg. P. Erzberger \& Cs. Németh (B-Erzberger 16007, dupl. hb. Németh). Balaton Uplands 9073.3 Comit. Veszprém. In rupibus basalticis umbrosis montis Kiserdőtető prope pag. Tihany, 24.06.1955. leg. L. Vajda (BP 39141), det. L. Vajda as Tortella viridiflava (De Not.) Broth., revised B. Papp June 2004 as T. tortuosa (Hedw.) Limpr. var. bambergeri (Schimp.) Duell. Comit. Veszprém (olim Zala) in graminosis saxosis declivium montis Hármas-hegy pr. pag. Tihany, 21.04.1957 leg. L. Felföldy, det. Á. Boros as Tortella tortuosa (Hedw.) Limpr. (BP 104017). 9173.1 Comit. Veszprém (olim Zala) in lapidibus calcareis umbrosis in silvis mixtis declivium montis Gurbicsa pr. pag. Tihany, 23.01.1956 leg. L. Felföldy, det. Á. Boros as Tortella tortuosa (Hedw.) Limpr. (BP 104015). Veszprém County. Tihany, Kerek-hegy, 13.02.2001 leg. P. Erzberger \& B. Papp (B-Erzberger 6755), det. Erzberger as Tortella cf. flavovirens (Bruch) Broth., revised P. Erzberger s.a. as T. bambergeri. Keszthely Mts $\mathbf{9 1 6 9 . 2}$ Comit. Zala. In rupestribus dolomit. adv. montem Rezi-vár prope Rezi, 3-400 m a.s.l., 23.03.1952 leg. Á. Boros as Tortella nitida (Lindb.) Broth. (BP 103972). 9169.3 Zala County, Cserszegtomaj, Csóka-kő, 27.06.2013 leg. P. Erzberger \& Cs. Németh (B-Erzberger 16416, dupl. hb. Németh). Köszeg Mts. 8664.2 Vas County, Kőszeg, between Kincs-pihenö and Szikla-forrás, on outcrops of

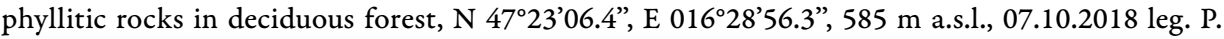
Erzberger \& K. Baráth (B-Erzberger 25583). Mecsek Mts 9776.4 Baranya County, east of Óbánya, west face of Templom-hegy (Szent-Imre-hegy), Fagetum nudum on steep slope with siliceous, but base-rich scree, $\mathrm{N} 46^{\circ} 13^{\prime} 08.2^{\prime \prime}$, E $018^{\circ} 25^{\prime} 56.5^{\prime \prime}, 310 \mathrm{~m}$ a.s.l., 29.03.2015 leg. P. Erzberger, J. Deme \& J. Csiky (B-Erzberger 19690). Geresdi-dombság 9777.4 Tolna County, south of Bátaapáti, broadleaved forest with granite rock outcrops, N 4612'03.3”, E 018³5'30.9”, $200 \mathrm{~m}$ a.sl., 12.10 .2015 leg. P. Erzberger, J. Deme, D. Kovács, J. Csiky \& I.-Zs. Tóth (B-Erzberger 20785).

\section{Tortella pseudofragilis (Thér.) Köckinger \& Hedenäs}

Aggtelek Karst 7490.1 Comit. Abaúj-Torna. In rupibus calcar. silvat. ad Vecsembükkizsomboly montis Vecsembükk prope Bódvaszilas, $550 \mathrm{~m}$ a.s.l., 02.11 .1937 leg. Á. Boros as Tortella tortuosa (Hedw.) Limpr. (BP 104183). Bükk Mts 7888.4 Heves County, ca $500 \mathrm{~m}$ north of Miskolc-Bánkút, on limestone rocks at the road, $\mathrm{N} 48^{\circ} 06^{\prime} 01.7^{\prime \prime}, \mathrm{E} 20^{\circ} 28^{\prime} 58.2^{\prime \prime}, 890 \mathrm{~m}$ a.s.l., 10.04 .2018 leg. S. Caspari \& P. Erzberger (B-Erzberger 24405, SAAR-GH7134), soc. Homalothecium philippeanum (Spruce) Schimp. 7889.3 Comit. Borsod. In rupibus calc.merid. Hetemér ad Szentlélek prope Ómassa, $650 \mathrm{~m}$ a.s.l., 06.11 .1957 leg. Á. Boros as Tortella nitida (Lindb.) Broth. (BP 103978; 103979 rev. by H. Reimers 29.04.1958 as Tortella tortuosa (Hedw.) Limpr). 7988.2 Comit. Borsod. In petrosis calcareis silvaticis montis Cserepeskő prope Szilvásvárad, $800 \mathrm{~m}$ a.s.l., 25.05 .1931 leg. Á. Boros as Tortella tortuosa (Hedw.) Limpr. (BP 104142). Comit. Borsod. In faginetis montis Kiskőhát prope Répáshuta, 800-900 m a.s.l., 05.06.1932 leg. Á. Boros as Tortella tortuosa (Hedw.) 
Limpr. (BP 104173). 7989.1 Borsod-Abaúj-Zemplén County, ca $4 \mathrm{~km}$ north-west of Répáshuta, in a dolina on the karst plateau, in crevices of limestone outcrops in mosaic of meadow and spruce forest, N 48 $04^{\prime} 50.3^{\prime \prime}$, E $20^{\circ} 30^{\prime} 14.3^{\prime \prime}, 780 \mathrm{~m}$ a.s.l., 09.04 .2018 leg. S. Caspari \& P. Erzberger (B-Erzberger 24387, 24390/A, SAAR-GH7135) soc. Schistidium robustum (Nees \& Hornsch.) H.H. Blom, S. dupretii (Thér.) W.A. Weber and S. elegantulum H.H. Blom.; also on small piece of limestone N 48 04'53.0”, E 20³0'34.8”, 770 m a.s.l., 09.04.2018 leg. S. Caspari \& P. Erzberger (B-Erzberger 24391, SAAR-GH7136). 7989.2 BAZ megye; Miskolc-Lillafüred; Szinva-völgy; árnyas mészkősziklán, $\mathrm{N} 48^{\circ} 05^{\prime} 08.8^{\prime \prime}, \mathrm{E} 020^{\circ} 36^{\prime} 52.8^{\prime \prime}, 350 \mathrm{~m}$ a.s.l., 17.03.2015 leg. Cs. Németh, P. Erzberger, B. Papp \& E. Szurdoki (hb. Németh 6532/5). This is the growth place of one of the largest Hungarian populations of Mannia triandra, a moderately cryophilic to moderately thermophilic species (DiersSEn 2001). 7989.4 Borsod-Abaúj-Zemplén County, ca $2.5 \mathrm{~km}$ west of Kisgyőr, north-east slope of the hill Pince-verö, on limestone boulder in deciduous forest, $\mathrm{N} 48^{\circ} 01^{\prime} 20.1^{\prime \prime}, \mathrm{E}$ $20^{\circ} 38^{\prime} 51.3$ ”, 440 m a.s.l., 15.04.2018 leg. P. Erzberger, J. Deme, D. Kovács \& J. Csiky (B-Erzberger 24529). Pilis Mts 8279.3 Komárom-Esztergom County, Pilisszentlélek, Fekete-kő (Hreben), shad-

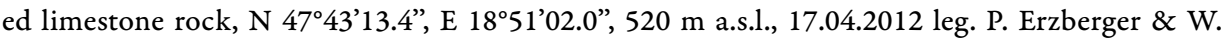
Wiehle (B-Erzberger 15227). 8379.1 Pest County, Pilis-Visegrád Mts, Pilistető hill at Pilisszent-

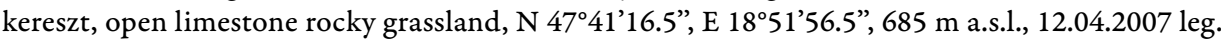
B. Papp as Tortella tortuosa (Hedw.) Limpr. (BP 174769). Pest County, Pilisszántó, summit of Pilishegy, calcareous grassland, deciduous forest with limestone outcrops and boulders, $\mathrm{N} 47^{\circ} 41^{\prime} 22.0^{\prime \prime}$, E 18 $51^{\prime}$ '57.6", 720 m a.s.l., 12.04.2007 leg. P. Erzberger \& B. Papp (B-Erzberger 12201). Bakony Mts 8872.2 Comit. Veszprém. In rupibus dolomit. silvat. montis Kisbükktető prope Márkó, $400 \mathrm{~m}$ a.s.l., 16.04.1967 leg Á. Boros as Tortella tortuosa (Hedw.) Limpr. (BP 104034). 